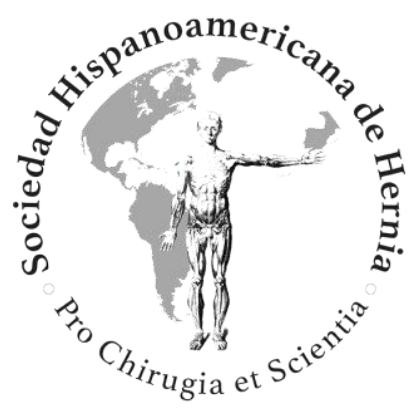

Eventración tras el cierre de la ileostomía de protección

\title{
Incisional hernia after loop ileostomy closure
}




\title{
Eventración tras el cierre de la ileostomía de protección Incisional hernia after loop ileostomy closure
}

\author{
Alba Vázquez Melero', Ventura Amador Barrameda², Aída Rico \\ Arrastia $^{3}$ \\ ${ }^{1}$ Hospital Universitario Araba. Vitoria (España). ${ }^{2}$ Hospital Insular \\ Nuestra Señora de Los Reyes. Villa de Valverde. Santa Cruz de \\ Tenerife (España). ${ }^{3}$ Complejo Hospitalario de Navarra. Pamplona \\ (España)
}

Autor para correspondencia: Alba Vázquez Melero. Hospital Universitario Araba. Jose Atxotegi Kalea, s/n. 01009 Vitoria (España) Correo electrónico: albavazquezmelero88@gmail.com

Recibido: 12-03-2020

Aceptado: 10-03-2020

\section{RESUMEN}

Introducción: La incidencia de la eventración en la localización previa de la ileostomía de protección alcanza el $48 \%$ de los casos en algunas series. Los factores de riesgo no han sido ampliamente estudiados.

Material y métodos: Se ha realizado un estudio de cohortes retrospectivo analizando todos los pacientes intervenidos de cierre de ileostomía de protección en el Hospital Universitario Basurto, de Bilbao (País Vasco, España), durante el periodo comprendido entre 2010 y 2018.

Resultados: Se analizaron un total de 134 pacientes a los que se les realizó cierre de la ileostomía temporal (57.46 \% hombres y $42.54 \%$ mujeres). La incidencia de eventración en la localización previa de la ileostomía fue del $11.19 \%$, con un seguimiento medio de entre 47 y 52 meses. Se apreció relación entre un IMC mayor a 30 y la presencia de eventración. 
Conclusiones: La incidencia de eventración en la localización previa de la ileostomía en nuestro estudio es moderada. La obesidad es el principal factor de riesgo.

\section{ABSTRACT}

Introduction: Incisional hernia at the stoma site after loop ileostomy reversal has a high incidence reaching $48 \%$ in some studies. Risk factors have not been largely studied.

Methods: This retrospective study includes all consecutive patients who underwent stoma reversal between 2010 and 2018 at the Department of General Surgery of the Basurto Hospital in Bilbao (Spain).

Results: A total of 134 patients ( $42.54 \%$ female, $57.46 \%$ male) had a stoma reversal operation. A postoperative incisional hernia at the stoma reversal site developed in $11.19 \%$ during a median follow-up of 47-52 months. Body mass index over 30, was associated with incisional formation at the stoma reversal.

Conclusion: Incisional hernia rate at the former stoma site in our study was moderated, obesity was the main risk factor.

Palabras clave: Cierre de ileostomía, eventración.

Keywords: Stoma closure, incisional hernia.

\section{INTRODUCCIÓN}

La ileostomía temporal o en asa se realiza tras intervenciones quirúrgicas como la resección anterior ultrabaja o la creación de anastomosis en el contexto de una peritonitis ${ }^{1}$. Se utiliza como estrategia para disminuir las consecuencias de la dehiscencia anastomótica al evitar el paso de heces por la misma, reduciendo la tasa de morbilidad y mortalidad secundaria a la sepsis ${ }^{2-4}$. Sin embargo, el restablecimiento del tránsito digestivo conlleva complicaciones como la eventración en la localización previa de la 
ileostomía, cuya incidencia es variable, ya que oscila entre el $0 \%$ y el $48 \% 5,6$.

El objetivo de este estudio consiste en establecer la incidencia de la eventración en la localización previa de la ileostomía, así como los factores de riesgo que favorecen su aparición.

\section{MATERIAL Y MÉTODOS}

\section{Pacientes}

Estudio de cohortes retrospectivo que analiza todos los pacientes intervenidos de cierre de ileostomía de protección en el Hospital Universitario Basurto, de Bilbao (País Vasco, España), durante el periodo comprendido entre febrero de 2010 y junio de 2018. Se registraron datos obtenidos tanto de la historia clínica en papel como de la digitalizada de las consultas durante el seguimiento de los pacientes tras la intervención.

\section{Variables analizadas}

- Se analizan aspectos demográficos como la edad, el sexo, el tabaquismo, el índice de masa corporal (IMC), la hipertensión arterial (HTA), la diabetes mellitus (DM), la cardiopatía o la neumopatía.

- En relación a la cirugía de creación de la ileostomía, se registra la indicación quirúrgica de la cirugía primaria, en la que se realiza la ileostomía (tumoral con ileostomía de manera profiláctica, tumoral con posoperatorio complicado, enfermedad inflamatoria u otras patologías), si la creación de la ileostomía se realiza de forma urgente o de manera programada y si se utiliza terapia adyuvante tras la misma.

- Respecto a la realización del cierre de la ileostomía, se observa si se realiza de forma precoz o tardía, el tipo de cierre de la piel (lineal o en bolsa de tabaco), si existe infección de la herida quirúrgica tras el mismo y la presencia de eventración. 
- En aquellos en los que se diagnostica la eventración, se analiza si esta se realiza mediante exploración física o pruebas complementarias, si se repara quirúrgicamente, el periodo de tiempo entre el cierre de la ileostomía y la reparación de la eventración del sitio quirúrgico y el tiempo de seguimiento.

Se define eventración, según la descripción de la European Hernia Society (EHS), como «cualquier defecto en la pared abdominal, acompañado o no de tumoración, en la misma localización de una cicatriz posoperatoria previa, perceptible mediante la exploración física o mediante pruebas de imagen». (7)

Su diagnóstico se realiza mediante la exploración física en bipedestación y en supino con maniobras de Valsalva, en la consulta del control posoperatorio, así como mediante la realización de tomografía computarizada (TAC) de control de la patología primaria (tumoral, inflamatoria...) (fig. 1).

\section{Análisis estadístico}

Los datos se recogen y se analizan mediante el paquete estadístico SPSS ${ }^{\circledR}$ Statistics V17.0. (SPSS, Chicago, Illinois, Estados Unidos) ${ }^{8}$. Las variables categóricas, como el género, la presencia de infección o la necesidad de adyuvancia, se describen como frecuencias absolutas o relativas y se analizan utilizando tablas de contingencia mediante el test de la $\chi^{2}$ de Pearson o con el test exacto de Fisher.

Las continuas, como la edad o el IMC, se describen como media y desviación estándar y se comparan utilizando la t de Student. Se considera un nivel estadístico de significación $(p)$ inferior a 0.05 .

\section{RESULTADOS}

Se analizan un total de 134 pacientes, a los que se les realiza cierre de la ileostomía temporal, 77 de los cuales fueron hombres $(57.46 \%)$ y 57 , mujeres (42.54\%), con una edad media de 62.81 (rango, 24-83 años). 
La indicación quirúrgica para la realización de la ileostomía fue de manera programada en el mismo acto quirúrgico que una resección anterior baja (RAB) en 100 pacientes (74.63\%), de urgencia tras complicaciones en la RAB sin ileostomía en 15 pacientes (11.20\%), debido a enfermedad inflamatoria en 8 pacientes (5.97\%) y por otras razones en 11 pacientes (8.20\%). Estos datos se muestran en la tabla I.

La cirugía primaria de creación de la ileostomía se programó en 113 pacientes $(84.33 \%)$ y fue de urgencia en 21 pacientes $(15.67 \%)$. Se observaron comorbilidades en 67 pacientes (50 \%). Las más frecuentes fueron la HTA y la diabetes mellitus (DM). En la tabla II están reflejadas las comorbilidades en los grupos con eventración y sin eventración.

Puede apreciarse que la obesidad supone un factor de riesgo para desarrollar eventración. Su relación es estadísticamente significativa $(p=0.002)$.

La incidencia de eventración en la localización previa de la ileostomía fue del $11.19 \%$, intervenida de media 21 meses tras la realización del cierre de la ileostomía.

Existió concordancia entre el diagnóstico clínico y el radiológico en el 80 \%; en 3 la exploración física no detectó la eventración, que sí fue objetivada radiológicamente.

Se intervino al $66.67 \%$ de los pacientes diagnosticados de eventración. De los casos restantes, 2 pacientes no quisieron operarse, 2 presentaban estadio IV del cáncer primario y 1 se encontraba pendiente de intervención.

En la tabla III se resumen las variables en relación a la cirugía de cierre de la ileostomía.

\section{DISCUSIÓN}

El cierre de la ileostomía de protección es una intervención quirúrgica muy frecuente. La incidencia de la eventración en la localización previa de la ileostomía varía mucho según los estudios revisados, ya 
que muchos incluyen eventraciones no solo tras el cierre de la ileostomía, sino también de la colostomía.

En la revisión sistemática de Bhangu y cols., la eventración tras el cierre de ostomía oscila entre el $0 \%$ y el $48 \%$. Sin embargo, la duración media del seguimiento de los pacientes en los estudios incluidos en la citada revisión sistemática es muy variable: entre 2 y 67 semanas $^{5}$. Es importante detectar los factores de riesgo para presentar eventración en la localización previa de la ileostomía para modificar preoperatoriamente aquellos que sea posible. Sin embargo, solo 2 de los 34 estudios incluidos en la revisión sistemática de Bhangu analizaron los posibles factores de riesgo, ${ }^{9,10}$. Por ello, en nuestro estudio retrospectivo de cohortes buscamos específicamente investigar la incidencia de la eventración en la localización previa de la ileostomía y los factores de riesgo que la aumentan.

Nuestros datos muestran una incidencia de eventración del $11.19 \%$. Los pacientes fueron intervenidos de media 21 meses después de la cirugía de cierre de la ileostomía.

Uno de los factores de riesgo relacionado con una mayor tasa de eventración es la obesidad, como se aprecia en el análisis retrospectivo de Zhou, en el que se observa una relación entre un mayor IMC y un aumento de la incidencia de eventración ${ }^{11}$. En concordancia con este estudio, nuestros resultados también apoyan que la obesidad es un factor de riesgo para desarrollar eventración del sitio quirúrgico ${ }^{12}$.

No hemos observado diferencias significativas en relación a la edad, el sexo, la DM o el tabaquismo. Estos resultados son parecidos a los obtenidos en el estudio prospectivo de Goodenough, que analizó 625 pacientes, con un seguimiento posoperatorio de 41 meses. Este estudio tampoco consiguió establecer una relación entre la edad, el sexo o el tabaquismo y la incidencia de la eventración ${ }^{13}$.

La influencia del tipo de cierre de la piel también se ha estudiado. En el artículo de Li, con 87 pacientes a los que se les realizó el cierre de la ileostomía de protección, la tasa de eventración en los pacientes 
con cierre de la piel en bolsa de tabaco no fue diferente a la de los pacientes con cierre lineal de la misma ${ }^{14}$. Sin embargo, el estudio de Juratli sí relaciona el cierre de la piel con bolsa de tabaco con una menor incidencia de eventración, probablemente por una menor infección de la herida quirúrgica ${ }^{15}$. Nuestro estudio, al igual que el de $\mathrm{Li}$, tampoco ha encontrado diferencias en la incidencia de la eventración según el tipo de cierre de piel.

Deberían utilizarse estrategias para reducir los factores de riesgo de desarrollar eventraciones en la localización previa de la ileostomía, como reducir el IMC o la colocación de mallas de manera profiláctica en la cirugía de cierre de la ileostomía ${ }^{17}$. En relación con este último, existen artículos que recomiendan el uso de estas mallas como profilaxis, como el de Liu, en el que se objetiva una tasa de eventración un $30 \%$ menor en el grupo con malla frente al grupo control, y el de Warren, con un $17.2 \%$ menos $^{18,19}$. También están realizándose varios ensayos aleatorizados al respecto, como el estudio aleatorizado multicéntrico ROCSS, de la Universidad de Birmingham (Reinforcement of Closure of Stoma Site, ClinicalTrials.gov NCT02238964), y el ILEOCLOSE (ClinicalTrials.gov NCT02226887), del Hospital Universitari Vall d'Hebron (Barcelona) ${ }^{20,21}$.

\section{CONCLUSIÓN}

En conclusión, la incidencia de eventración en la localización previa de la ileostomía en nuestro estudio es moderada (del $11.19 \%$ ) y la obesidad, el principal factor de riesgo. Estrategias de reducción del IMC en pacientes obesos de manera preoperatoria podrían reducir la tasa de eventración.

\section{BIBLIOGRAFÍA}

1. Thoker M, Wani I, Parray F, et al. Role of diversion ileostomy in low rectal cancer: A randomized controlled trial. Int J Surg. 2014;12:945-51. DOI: 10.1016/j.ijsu.2014.07.012 
2. Hüser N, Michalski C, Erkan M. Systematic review and metaanalysis of the role of defunctioning stoma in low rectal cancer surgery. Ann Surg. 2008;248:52-60. DOI: 10.1097/SLA.0b013e318176bf65

3. Tan W, Tang C, Shi L, et al. Meta-analysis of defunctioning stomas in low anterior resection for rectal cancer. $\mathrm{Br} J$ Surg. 2009;96:462-72. DOI: 10.1002/bjs.6594

4. Chen J, Wang D, Yu H, et al. Defunctioning stoma in low anterior resection for rectal cancer: a meta-analysis of five recent studies. Hepatogastroenterol. 2012;59:1828-31.

5. Bhangu A, Nepogodiev D, Futaba K; West Midlands Research Collaborative. Systematic review and meta-analysis of the incidence of incisional hernia at the site of stoma closure. World J Surg. 2012;36(5):973-83. DOI: 10.1007/s00268-012-1474-7

6. Brook A, Mansfield S, Daniels I, et al. Incisional hernia following closure of loop ileostomy: The main predictor is the patient, not the surgeon. Surgeon. 2018;16(1):20-6. DOI: 10.1016/j.surge.2016.03.004

7. Muysoms F, Deerenberg E, Peeters E, et al. Recommendations for reporting outcome results in abdominal wall repair: results of a Consensus meeting in Palermo, Italy, 28-30. Hernia J Hernias Abdom Wall Surg. 2013;17:423e33. DOI: 10.1007/s10029-0131108-5

8. SPSS Inc. SPSS for Windows. Chicago: SPSS Inc; 2008.

9. Guzmán-Valdivia G. Incisional hernia at the site of a stoma. Hernia. 2008;12(5):471-4. DOI: 10.1007/s10029-008-0378-9

10. Cingi A, Cakir T, Sever A, et al. Enterostomy site hernias: a clinical and computerized tomographic evaluation. Colon Rectum. 2006;49(10):1559-63. DOI: 10.1007/s10350-006-06814

11. Zhou P, Hrabe J, Byrn J. A retrospective, single-institution review of loop ileostomy reversal outcomes. Ostomy Wound Manage. 2016;62(8):22-33. 
12. Itatsu K, Yokoyama Y, Sugawara G, et al. Incidence of and risk factors for incisional hernia after abdominal surgery. Br J Surg. 2014;101:1439e47. DOI: 10.1002/bjs.9600

13. Goodenough C, Ko T, Kao L, et al. Development and validation of a risk stratification score for ventral incisional hernia after abdominal surgery: hernia expectation rates in intraabdominal surgery (the HERNIA project). J Am Coll Surg. 2015;220:405e13. DOI: 10.1016/j.jamcollsurg.2014.12.027

14. Li L, Brahmbhatt R, Hicks S, et al. Prevalence of surgical site infection at the stoma site following four skin closure techniques: a retrospective cohort study. Dig Surg. 2014;31:73e8. DOI: 10.1159/000354426

15.Juratli M, Nour-Eldin N, Ackermann $\mathrm{H}$, et al. Purse-string closure technique reduces the incidence of incisional hernias following the reversal of temporary ileostomy. Int J Colorectal Dis. 2018;33(7):973-7. DOI: 10.1007/s00384-018-2986-x

16. Sanders $D$, Kingsnorth $A$. The modern management of incisional hernias. BMJ Clin Res Ed. 2012;344:e2843. DOI: 10.1136/bmj.e2843

17. Hernández-Granados P, López-Cano M, Morales-Conde S, et al. Incisional hernia prevention and use of mesh. A narrative review. Cir Esp. 2018;96(2):76-87. DOI: 10.1016/j.ciresp.2018.01.003

18. Liu D, Banham E, Yellapu S. Prophylactic mesh reinforcement reduces stomal site incisional hernia after ileostomy closure. World J Surg. 2013;37(9):2039-45. DOI: 10.1007/s00268-0132109-3

19. Warren J, Beffa L, Carbonell A, et al. Prophylactic placement of permanent synthetic mesh at the time of ostomy closure prevents formation of incisional hernias. Surgery. 2018;163(4):839-46. DOI: 10.1016/j.surg.2017.09.041

20. Reinforcement of Closure of Stoma Site (ROCSS) Collaborative and the West Midlands Research Collaborative. Randomized 
controlled trial of standard closure of a stoma site vs biological mesh reinforcement: study protocol of the ROCSS trial. Colorectal Dis. 2018;20(2):46-54. DOI: 10.1111/codi.13997

21. Villanueva B, Vallribera F, López Cano M. Prophylaxis of ileostomy closure site hernia by placing mesh (ILEOCLOSE). ClinicalTrials.gov.

Tabla I. Indicación quirúrgica para la creación de la ileostomía

\begin{tabular}{|l|l|}
\hline Indicación quirúrgica & Número (\%) \\
\hline RAB programada & $100(74.63 \%)$ \\
Complicación tras RAB & $15(11.20 \%)$ \\
Enfermedad inflamatoria & $8(5.97 \%)$ \\
Otras & $11(8.20 \%)$ \\
Total & $134(100 \%)$ \\
\hline
\end{tabular}

Tabla II. Características demográficas y comorbilidades

\begin{tabular}{|c|c|c|c|}
\hline & $\begin{array}{l}\text { Con } \\
\text { eventración }\end{array}$ & $\begin{array}{l}\text { Sin } \\
\text { eventración }\end{array}$ & $\mathbf{p}$ \\
\hline Pacientes & $\mathrm{n}=15(11.19$ & $\mathrm{n}=119$ & - \\
\hline Edad media, años & $\begin{array}{l}\%) \\
64.27(42-78)\end{array}$ & $\begin{array}{l}\%) \\
62.62(24-83)\end{array}$ & 0.613 \\
\hline $\begin{array}{l}\text { (rango) } \\
\text { Hombres: mujeres }\end{array}$ & $1.5: 1$ & $1.3: 1$ & - \\
\hline (ratio) & & & \\
\hline $\begin{array}{l}\text { Fumadores } \\
\text { IMC (rango) }\end{array}$ & $\begin{array}{l}1(7.00 \%) \\
28(19-40)\end{array}$ & $\begin{array}{l}22(18.49 \%) \\
26(17-36)\end{array}$ & $\begin{array}{l}0.253 \\
0.895\end{array}$ \\
\hline Obesidad & $6(40.00 \%)$ & $13(10.92 \%)$ & 0.002 \\
\hline Adyuvancia & $4(26.67 \%)$ & $54(45.38 \%)$ & 0.168 \\
\hline Comorbilidades & $11(73.33 \%)$ & 56 (47.06 \%) & 0.055 \\
\hline DM & $4(26.67 \%)$ & $16(13.44 \%)$ & 0.176 \\
\hline HTA & $8(53.33 \%)$ & 45 (37.81\%) & 0.247 \\
\hline Neumopatía & $2(13.33 \%)$ & $7(5.88 \%)$ & 0.277 \\
\hline Cardiopatía & $2(13.33 \%)$ & $6(5.04 \%)$ & 0.202 \\
\hline
\end{tabular}


Tabla III. Variables en relación a la cirugía de cierre de la ileostomía

\begin{tabular}{|c|c|c|c|}
\hline & $\begin{array}{l}\text { Con } \\
\text { eventración }\end{array}$ & $\begin{array}{l}\text { Sin } \\
\text { eventración }\end{array}$ & $\mathbf{p}$ \\
\hline Pacientes & $\mathrm{n}=15(11.19 \%)$ & $\mathrm{n}=119(88.81$ & - \\
\hline $\begin{array}{l}\text { Cierre precoz } 1<90 \\
\text { días })\end{array}$ & $9(60.00 \%)$ & $\begin{array}{l}\%) \\
44(36.98 \%)\end{array}$ & 0.086 \\
\hline Cierre piel (Lineal) & $12(80.00 \%)$ & $83(69.78 \%)$ & 0.410 \\
\hline Infección de la herida & $5(33.33 \%)$ & $24(20.17 \%)$ & 0.243 \\
\hline Diagnóstico clínico & $12(80.00 \%)$ & - & \\
\hline $\begin{array}{l}\text { Diagnóstico radiológico } \\
\text { Concordancia }\end{array}$ & $\begin{array}{l}15(100 \%) \\
80 \%\end{array}$ & - & \\
\hline $\begin{array}{l}\text { diagnóstica } \\
\text { Eventraciones }\end{array}$ & $10(66.67 \%)$ & - & - \\
\hline $\begin{array}{l}\text { intervenidas } \\
\text { Tiempo } \\
\text { intervención }\end{array}$ & $21(10-62)$ & & - \\
\hline $\begin{array}{l}\text { (meses) (rango) } \\
\text { Tiempo de seguimiento } \\
\text { (meses) (rango) }\end{array}$ & $52(26-102)$ & $47(5-118)$ & 0.416 \\
\hline
\end{tabular}

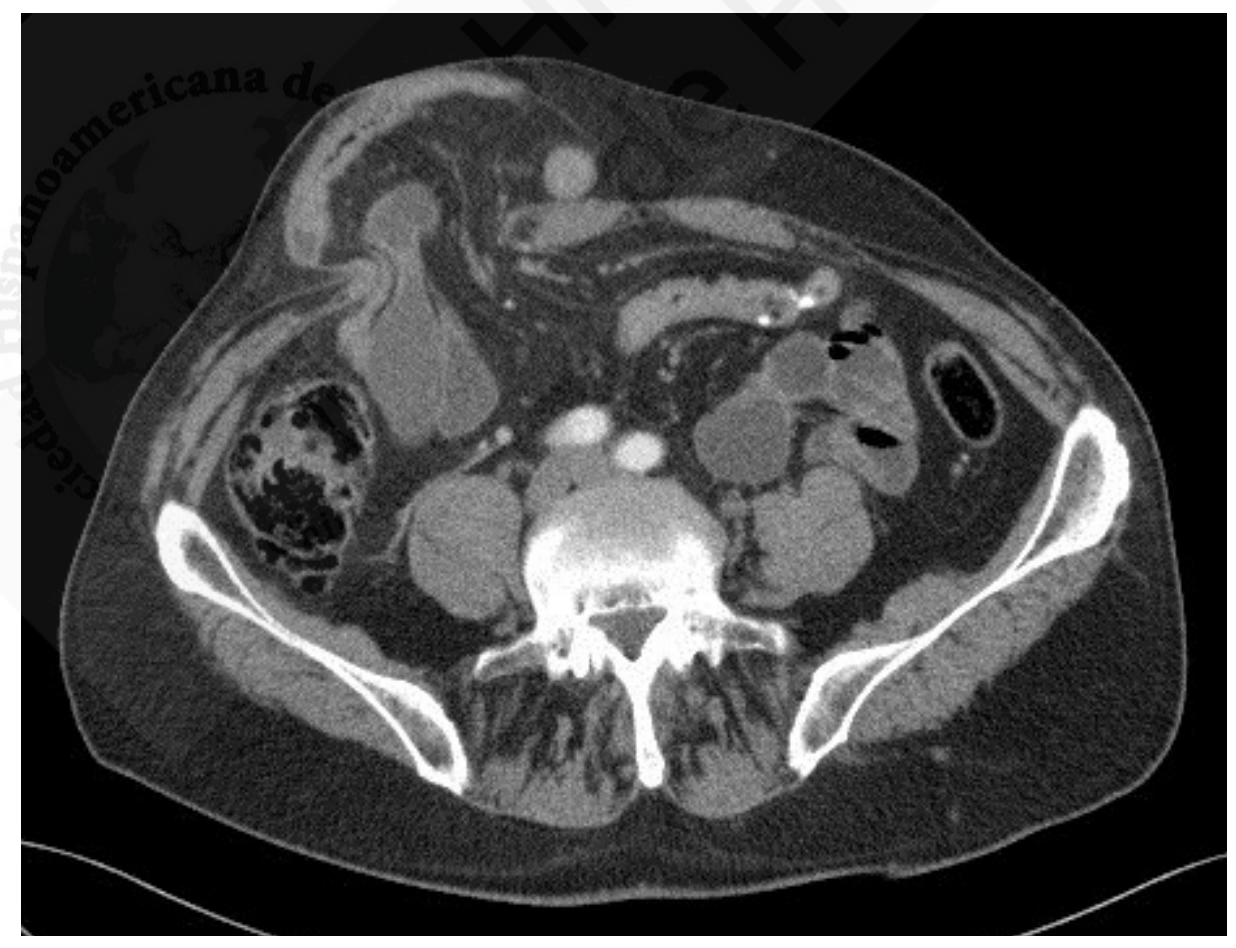


Figura 1. Eventración en la localización previa de la ileostomía. 